\title{
Effects of Riparian Vegetation on the Transport and Accumulation of Floating Objects in a Meandering River
}

\author{
Nakayama Akihiko ${ }^{1}$, Huan Tao Goh ${ }^{1 *}$ and Seak Ni Chai ${ }^{1}$ \\ ${ }^{1}$ Faculty of Engineering and Green Technology, Universiti Tunku Abdul Rahman, Jalan Universiti, \\ Bandar Barat, 31900 Kampar, Perak, Malaysia
}

\begin{abstract}
In order to study and understand the characteristics of transport and depositions of floating objects in real rivers, Large Eddy Simulation (LES) method of real river flows with complex bathymetry, the riparian vegetation and the floating objects has been developed and applied to a meandering river in Perak, Malaysia. The movement and accumulation of floating objects are different for different sizes and the shapes of the objects. The vegetation that may exists on the bed and the banks also are seen to influence the positions where the objects are accumulated and deposited. The results can be used to control the increasing amount of solid objects washed into the rivers and to the ocean.
\end{abstract}

\section{Introduction}

Rivers transport various objects mostly from upstream to the downstream and into the sea as the water flows. In the natural processes such as the sediment and the debris of trees and vegetation are the most common substances that are parts of the natural cycle keeping the supply of sand on the beaches and nutrients for fish and aquatic organisms. Recently, however, human produced objects from household and industrial waste are increasing creating sources of environmental pollution. Large amount of trash is found accumulating in certain places in the oceans and much of them appear to come from the rivers. They not only influence the aesthetic and other qualities of the natural and constructed streams but can also be a source of nonreversible global pollution. Large objects such as wood branches and debris that are washed into flows can not only influence the conveyance of the river streams but can damage structures like bridges and hydropower intake structures [1]. Management of these floating objects in rivers is becoming a major issue in developed and developing countries [2].

In order to control the movement and accumulation of discarded objects, the present authors simulated the transport and accumulation of floating objects in a large river in Malaysia [3]. The motion of floating objects in rivers is limited by the banks and they mostly follow the river flow itself. However, the complex plan and cross sectional

\footnotetext{
* Corresponding author: gohhuantao@gmail.com
} 
geometry of the rivers make the transport of the floating objects complex as well. It was found that the small objects tend to follow the surface flow but large ones with directional characteristics can move quite differently. In meandering rivers with complex topography, objects of different sizes and the shapes are found to be transported, accumulated and deposited at different locations of the rivers.

In the above simulation study, while the complex bed and bank geometries are represented, the vegetation and bushes that grow in the downstream reaches of the river are not accounted for. The secondary flows in meandering river reach interact with the vegetation [4-6], so the motion of the floating objects will also be influenced. In the present work, the vegetation that grow on the bed and the banks are modeled by in-flow resistance and their effects on the main flow, the secondary flows and the motion of the floating objects are reflected in the simulation. The results are compared and the estimates in the real river in Malaysia are discussed.

\section{Basic equations and calculation method}

\subsection{Calculation of the flow}

The flow of the river is calculated using a finite difference method constructed on rectangular grid on Cartesian coordinates. The flow regions in natural rivers are very irregular in shape and change in time and the fixed rectangular grid is more suited than boundary-following curvilinear coordinates. Using the rectangular grid it is relatively easy to represent flow changing regions due to increasing or decreasing flow rate. The free surface is treated as a dynamically moving surface. The turbulence and the small scale variations in the boundaries and the water surface are modeled in the generalized Large Eddy Simulation (LES) in which the governing equations are the three-dimensional unsteady equations of motion but filtered at the scale that can be resolved by the numerical grid and that the un-resolvable small scale variations are represented by sub-grid scale models. The overall method follows the method used by a present author [7].

The filtered equations of motion and the continuity equation for the spatially-filtered velocity components $(u, v, w)$ and the filtered pressure $p$ in rectangular coordinates $(x, y, z)$ with $z$ taken positive vertically upward are

$$
\begin{gathered}
\frac{\partial u}{\partial t}+\frac{\partial u^{2}}{\partial x}+\frac{\partial u v}{\partial y}+\frac{\partial u w}{\partial z}=-\frac{1}{\rho} \frac{\partial p}{\partial x}+\frac{\partial}{\partial x}\left(v \frac{\partial u}{\partial x}+\tau_{x x}\right)+\frac{\partial}{\partial y}\left(v \frac{\partial u}{\partial y}+\tau_{y x}\right)+\frac{\partial}{\partial z}\left(v \frac{\partial u}{\partial x}+\tau_{z x}\right) \\
\frac{\partial v}{\partial t}+\frac{\partial u v}{\partial x}+\frac{\partial v^{2}}{\partial y}+\frac{\partial v w}{\partial z}=-\frac{1}{\rho} \frac{\partial p}{\partial y}+\frac{\partial}{\partial x}\left(v \frac{\partial v}{\partial x}+\tau_{x y}\right)+\frac{\partial}{\partial y}\left(v \frac{\partial v}{\partial y}+\tau_{y y}\right)+\frac{\partial}{\partial z}\left(v \frac{\partial v}{\partial x}+\tau_{z y}\right) \\
\frac{\partial w}{\partial t}+\frac{\partial u w}{\partial x}+\frac{\partial v w}{\partial y}+\frac{\partial w^{2}}{\partial z}=-\frac{1}{\rho} \frac{\partial p}{\partial z}-g+\frac{\partial}{\partial x}\left(v \frac{\partial w}{\partial x}+\tau_{x z}\right)+\frac{\partial}{\partial y}\left(v \frac{\partial w}{\partial y}+\tau_{y z}\right)+\frac{\partial}{\partial z}\left(v \frac{\partial w}{\partial z}+\tau_{z z}\right)
\end{gathered}
$$

and

$$
\frac{\partial u}{\partial x}+\frac{\partial v}{\partial y}+\frac{\partial w}{\partial z}=0
$$

where $t$ is time, $\rho$ and $v$ are the density and the kinematic viscosity of water, $g$ is the gravitational acceleration, and $\tau_{x x}$ etc. are the effective stress components representing the effects of the unresolved scales of motion.

The free-surface position is given by the elevation $h$ of the filtered water surface along with the velocity field. If $h$ is a single-valued function of the horizontal position $(x, y)$, it is 


$$
\frac{\partial h}{\partial t}+\frac{\partial u_{s} h}{\partial x}+\frac{\partial v_{s} h}{\partial y}=w_{s}+\frac{\partial}{\partial x} \tau_{h x}+\frac{\partial}{\partial y} \tau_{h y}
$$

where $u_{s}, v_{s}, w_{s}$ are the (filtered) velocity components at the water surface, and $\tau_{h x}$ and $\tau_{h y}$ are the sub-grid scale free surface fluctuation terms [8].

For the boundary condition on the river bed, the generalized rough-surface wall model in which the bed shear stress is determined from the velocity at the calculation point closest to the bed using the general velocity similarity law that includes the laminar and smooth flow depending on the local Reynolds roughness number. The inflow velocity and pressure distributions are calculated by setting a driver region where the distribution at the downstream section of this development region is fed back to the inflow section as done by [9] for simulation of turbulent boundary layers. For the downstream outflow condition, the uniform flow condition is applied and the flow depth is given from the local flow rate assuming the flow is uniform with the assumed bed roughness coefficient. Therefore the location of the downstream section is chosen so that the non-uniform effects are minimal.

\subsection{Dynamic model for vegetation effects}

The effects of vegetation in the river or channel flows have been investigated by many researchers. The common way of modelling is to consider spatial averaged effects [10], spatial averaging is being used [11] which is most suited for the LES based methods. In the present simulation, although the flow depth can be resolved quite small to represent the depth-wise variation of vegetation growth, the horizontal dimensions of individual vegetation are smaller than typical horizontal grid spacing, which can be as large as $10 \mathrm{~m}$ for large rivers with width of several hundred meters. Therefore the effects of the vegetation on the flow are represented as inflow resistance. Figure 1 shows typical situation of vegetation within flow. The resistance $\boldsymbol{D}$ is distributed in the flow by

$$
\boldsymbol{D}=-\frac{1}{2} C_{D v}(z) \Lambda V_{f} \boldsymbol{V}_{\boldsymbol{f}}
$$

where $C_{D v}$ is the drag coefficient of which value is determined for different types of vegetation, $\Lambda$ is the leave-area density and $\boldsymbol{V}_{f}$ is the local flow velocity $(u, v, w)$. The values

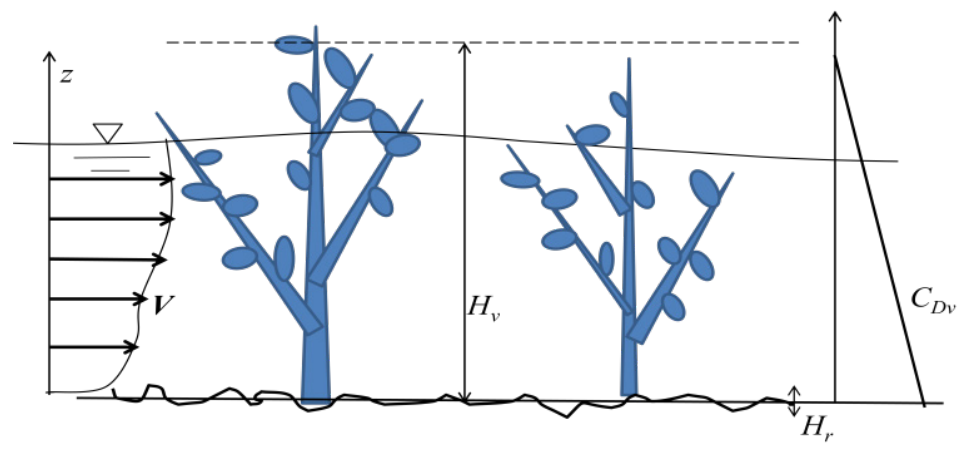

Fig. 1. Vegetation model in terms of distributed drag coefficient.

of $C_{D v}$ is assumed to vary linearly with the height from the bed to the top of the vegetation taken to be are 


$$
\boldsymbol{D}=-\frac{1}{2} C_{D v}(z) \Lambda V_{f} \boldsymbol{V}_{\boldsymbol{f}}
$$

with the $C_{D 0}$ the maximum value at the bottom.

The bed roughness is separately set in the wall boundary conditions for the velocity.

The equivalent height $H_{r}$ is set depending on the bed morphology and the materials.

\subsection{Equations for the motion of floating objects}

The motion of floating objects is receiving attention after recent large floods have shown that large debris including drift woods can cause serious damages to the river structures and also creating disruption of smooth flow of water [12]. Studies of modeling the motion of these objects are also conducted by a few researchers [13] and a few different models are proposed [14-15].

In the present work of computing the motion of floating objects, it is assumed that the specific weight of the objects is not too heavy so that they float on the water surface all the time. The vertical component of the hydrodynamic force and its moments about horizontal axes are assumed small so the submerged volume of the object stays unchanged and the motion is in the plane of the free surface. Therefore the velocity components only in the horizontal directions are computed from the equations of motion. Also the size of the objects is small compared with the grid size used to calculate the flow. This means that the calculated flow around the body is almost uniform. So the force due to the flow can be parameterized by the local flow velocity. Figure 2(a) depicts the body moving with three degrees of freedom in the horizontal $(x, y)$ plane on the free surface. The coordinates $(x, y)$ are fixed in space and coordinates $\left(x_{b}, y_{b}\right)$ are fixed on the moving body also horizontal. $\boldsymbol{V}_{f}$ $=(u, v)$ is the velocity of water in the vicinity of the floating body on the free surface and $\boldsymbol{V}_{b}$ $=\left(u_{b}, v_{b}\right)$ is the translation velocity and $\omega_{b}$ is the angular velocity of the floating body.

(a)

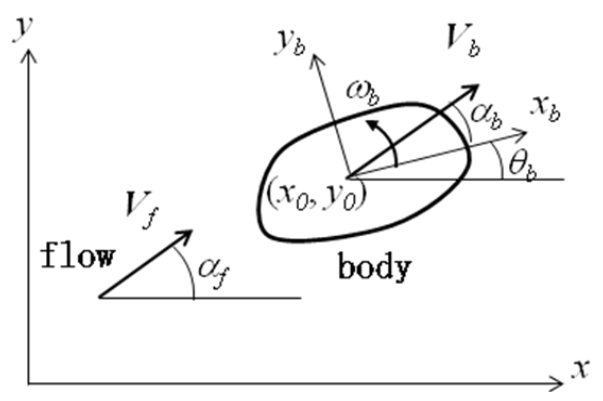

(b)

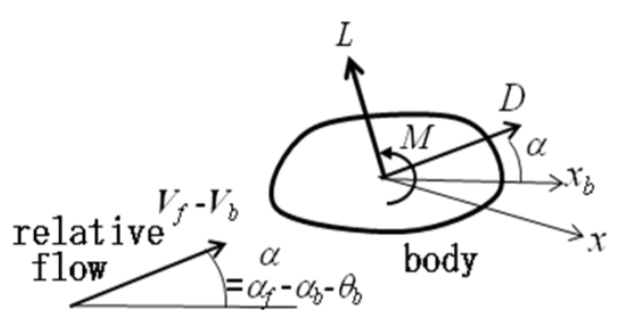

Fig. 2. Motion of floating body and the forces acting on it.

Figure 2(b) shows the forces acting on the body. $D$ is the component of the fluid force in the direction of the flow relative to the moving body. $L$ is the component of the fluid force perpendicular to the relative flow direction in the plane of the free surface. The positive $L$ is taken so that the flow direction, the direction of the positive $L$ and the vertically upward direction form the right-hand coordinate axes. $D, L$ and $M$ are evaluated from the usual definition of the drag, lift and yawing moment coefficients, $C_{D}, C_{L}$ and $C_{M}$ as follows. 


$$
\begin{aligned}
D & =\frac{1}{2} C_{D}(\alpha) A_{x} \rho\left|V_{f}-V_{b}\right|^{2}, \\
L & =\frac{1}{2} C_{L}(\alpha) A_{y} \rho\left|V_{f}-V_{b}\right|^{2}, \\
M & =\frac{1}{2} C_{D M}(\alpha) A_{y} \rho\left|V_{f}-V_{b}\right|^{2}
\end{aligned}
$$

where $\alpha$ is the 'angle of attack' of the relative flow measured from the direction of the body axis $\theta_{b}$,

$$
\alpha=\alpha_{f}-\alpha_{b}-\theta_{b}
$$

$A_{x}$ and $A_{y}$ are the cross sectional areas of the submerged part of the body in the axial and transverse directions and $l_{x}$ is the length of the body.

The equations of motion for the body are

$$
\begin{gathered}
m_{b} \frac{d u_{b}}{d t}=D \cos d \alpha-L \sin d \alpha \\
m_{b} \frac{d v_{b}}{d t}=D \sin d \alpha+L \cos d \\
I_{b} \frac{d \omega_{b}}{d t}=M
\end{gathered}
$$

where $d \alpha=\alpha_{f}-\alpha_{b}, m_{b}$ is the mass of the body and $I_{b}$ is the moment of inertia of the horizontal cross section of the body. The position $\left(x_{0}, y_{0}\right)$ and the angle $\theta_{b}$ of orientation of the body are then obtained by integrating the respective velocities with time. This method will be a good approximation if the non-uniformity of the flow is of larger scale than the size of the bodies.

The equations of motion of the floating objects are solved by the explicit differencing. The motion of the bodies is much slower than the time scale of the flow calculation and the low order time integration is sufficient. In the present computation, the time step of the flow calculation is $0.005 \mathrm{~s}$ and the time scale of the drag forces on bodies with size $1 \mathrm{~m}$ is close to $1 \mathrm{~s}$.

\section{River reach considered}

Calculation is conducted for meandering river flow in a low land in Perak, Malaysia. Figure 3 shows the meandering reach of Perak River near the town of Teluk Intan in Perak State. Perak River meanders around before it empties into Melaka Strait and the bends in Teluk Intan are very acute. A tributary flowing in and some bars near the inner banks make the motion of floating objects very complex. The radius of the curve is only about two times the river width and the effects of the bend are expected to be very strong [15].

The bathymetry and the areas of vegetation are shown in Figure 4(b). This map of elevation of the bed and the bank has been constructed based on the data obtained from the Department of Irrigation and Drainage Department of Malaysia. The average bed roughness without considering the vegetation is taken as $0.8 \mathrm{~cm}$. This includes the effects of the bed undulation and the sediment size at this section of the river. 
(a) Google satellite image

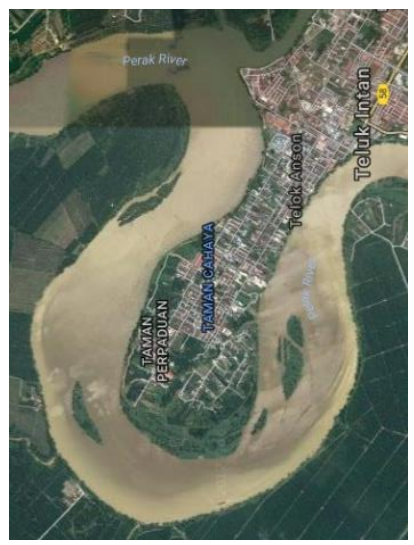

(b) elevation contours and vegetation regions (Interval between contour is $0.5 \mathrm{~m}$ )

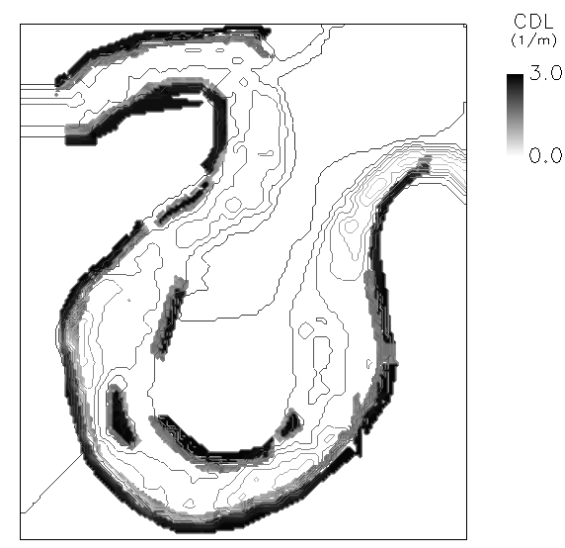

Fig. 3. Perak River in Teluk Intan.

\section{Simulation results}

A typical result of the mean flow calculation by the LES is shown in Figure 4. It shows the velocity vectors on the free surface of the time averaged flow obtained by a simulation with fixed discharge rate of $2600 \mathrm{~m}^{3} / \mathrm{s}$ and $1000 \mathrm{~m}^{3} / \mathrm{s}$. The average slope of the river surface in the area is about $1 / 2000$.

The bed roughness in terms of the equivalent sand roughness is $0.02 \mathrm{~m}$. In each of these cases the computational grid is a fixed rectangular mesh of approximately $200 \times 200 \mathrm{x}$ 60 points with horizontal and vertical spacings of $20 \mathrm{~m}$ and $0.4 \mathrm{~m}$, respectively. Flow calculation is started with an assumed initial flow of assumed logarithmic vertical distribution.

(a) Discharge $2600 \mathrm{~m}^{3} / \mathrm{s}$

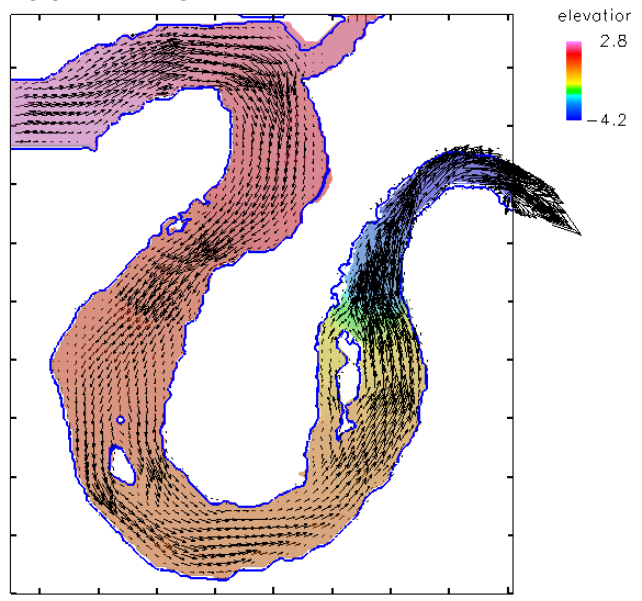

(b) Discharge $1000 \mathrm{~m}^{3} / \mathrm{s}$

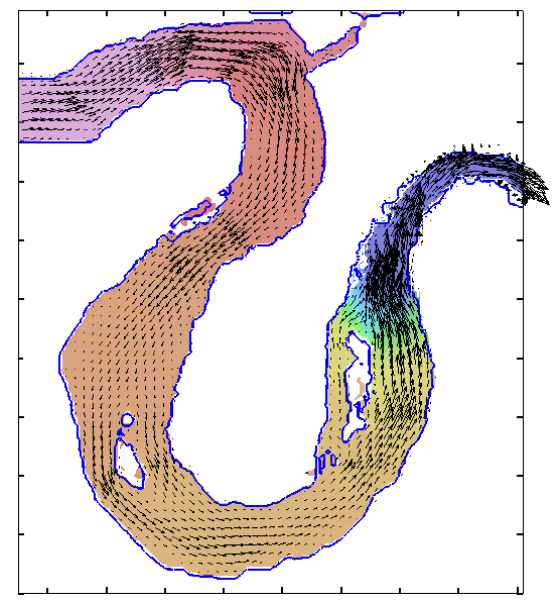

Fig. 4. Surface elevtion and the velocity distributions. 
For examining the motion of floating objects, about 110 bodies are first placed at the inflow section at $x=0 \mathrm{~m}$ of the present simulation region. Then as the flow is computed, the motion of these floating objects is tracked. Two types of objects are released. The first one is small objects with negligible mass and the second one with cylindrical shape of length $2 \mathrm{~m}$ and the diameter $0.2 \mathrm{~m}$. It is a typical tree trunk that is often found in this river. The drag coefficient is assumed to be 1.0 and the yaw moment coefficient is assumed to be -0.4 which is a typical shape with stability for rotation [16].

Figure 5 shows the positions of small objects with negligible mass at 2000s, 4000s and 6000 s after they are released at the inflow section. These are the results for the discharge of $1000 \mathrm{~m}^{3} / \mathrm{s}$.

(a) $t=2000 \mathrm{~s}$, small objects, without vegetation

(b) $t=2000 \mathrm{~s}$, small objects, with

vegetation

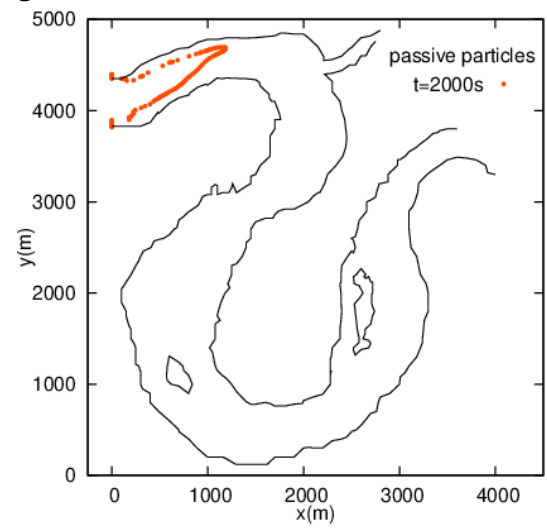

(c) $t=4000 \mathrm{~s}$, small objects, without vegetation vegetation

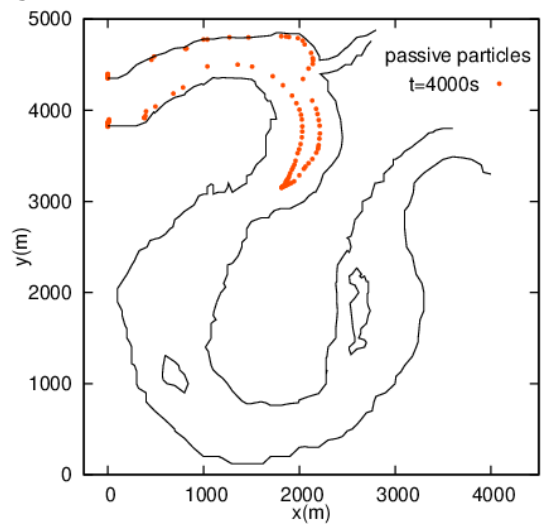

(e) $t=6000 \mathrm{~s}$, small objects, without vegetation vegetation

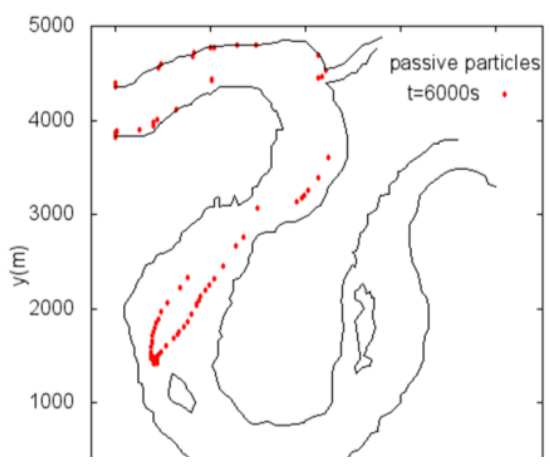

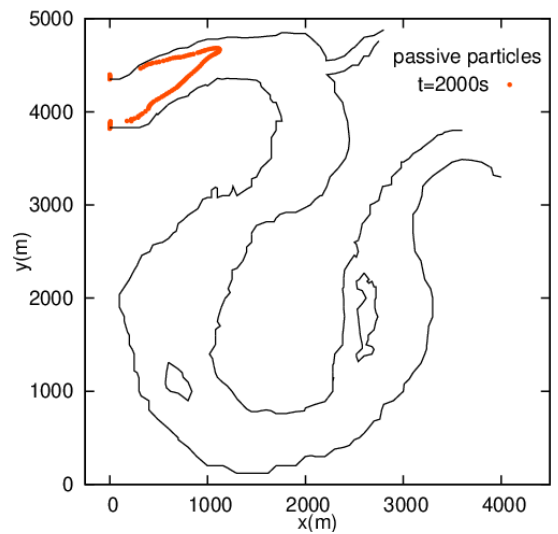

(d) $t=4000 \mathrm{~s}$, small objects, with

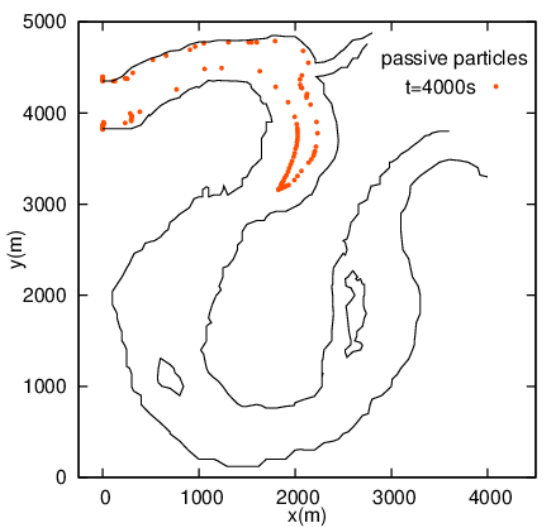

(f) $t=6000 s$, small objects, with

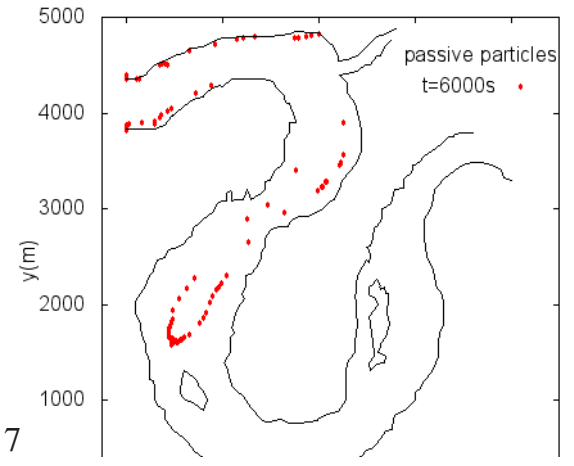


Fig. 5. Positions of small objects after released across the width at the upstream inflow plane with spacing of $5 \mathrm{~m}$ between objects.

(a) $t=4000 \mathrm{~s}$, long bodies, without vegetation

(b) $t=4000$ s, long bodies, with

\section{vegetation}

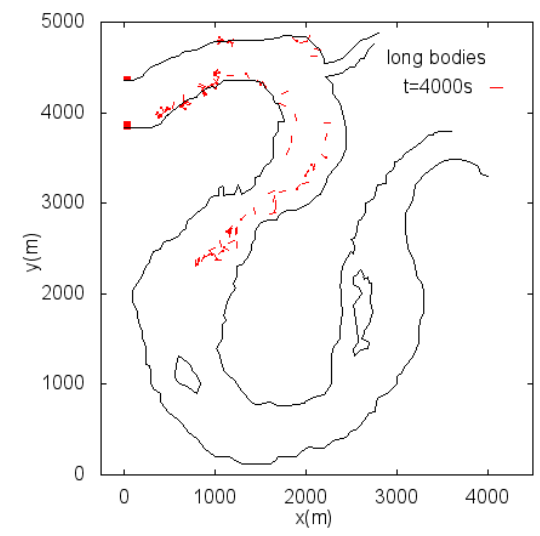

(c) $t=6000 \mathrm{~s}$, long bodies, without vegetation vegetation

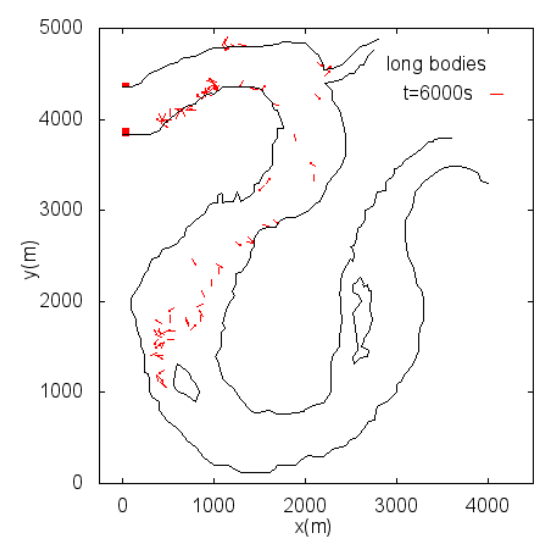

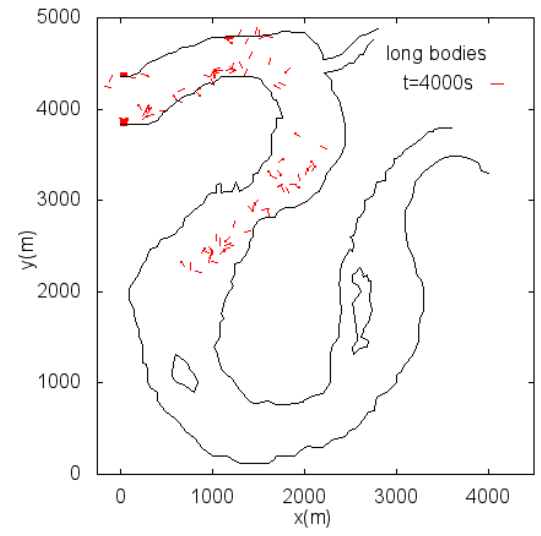

(d) $t=6000$, long bodies, with

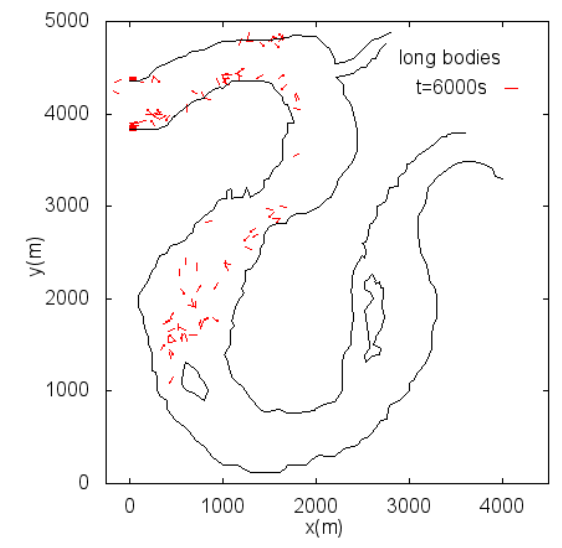

Fig. 6. Positions of long objects of length $2 \mathrm{~m}$ and diameter $0.2 \mathrm{~m}, 4000 \mathrm{~s}$ and $6000 \mathrm{~s}$ after released across the width at the upstream inflow plane with spacing of $10 \mathrm{~m}$ between objects.

Figures 5(a), (c) and (e) show the results with no vegetation and Figures 5(b), (d) and (f) when there are vegetation patches along the banks as shown in Figure 3. Since the particles in these cases are assumed very small they indicate the Lagrangian trajectories of the surface flow. It appears that there is retardation when the objects are in the vegetated regions. The particles tend to approach the banks as they travel downstream and they cling to the banks as the velocity becomes zero. The characteristics are consistent with the earlier simulation done for higher discharge rate.

Figure 6 shows the movement of long objects 4000s and 6000s after the release. Since they have stable pitching coefficient they tend to move without rotation in flows without secondary flows or vegetation but when the flow itself turns these long bodies tend to rotate as well and depending on the angle they can move fast or slow. Especially where the vegetation creates the lateral shear they tend to rotate and disperse rapidly. The 
transportation characteristics of the long bodies appear to be strongly influenced by vegetation near the banks. Also they are seen to accumulate near the banks like the massles passive particles.

\section{Discussion}

From Figure 4, the calculations results show that in the case of flow rate $2600 \mathrm{~m}^{3} / \mathrm{s}$ indicate higher magnitude of velocity than the lower flow rate of $1000 \mathrm{~m}^{3} / \mathrm{s}$. The result from Figure 4 also indicates that several factors influence the magnitude of velocity. They are curvature of the river, width of the river, and the vegetation. Significant increase of the velocity is seen near the bend and the narrow parts of the river. It also show the correlation between velocity and the local depth of the river, whereby the riverbed is deeper in the area with higher velocity. The phenomena that is observed from figure 4 can be explained with Donatella Termini's works. In her work, result shows the correlation between the flow characteristic and width to depth ratio. Degree of an overlap of circulation cells at the crossover increases as the width-to-depth ratio decreases [15]. Furthermore, in Donatella's work aforementioned conclusion that core of high velocities will shift outwards as the channel curvature increases [15]. This conclusion also was made in a previous work [17].

Results from Figure 5 and Figure 6 elaborate the movement of floating objects in the conditions with and without vegetation. Different floating objects are assumed in the simulation and the movement of small objects with negligible mass are shown in Figure 5 while long bodies with cylindrical shape are shown in Figure 6.

In Figure 5, the movement of the particles are seen to be not much difference between the conditions with and without vegetation. Still, there is a subtle difference where the floating objects tend to accumulate and cling near the riverbank in the case without vegetation. While in Figure 6, the difference between the condition with and without vegetation is clearer.

Floating long bodies in Figure 6 (without vegetation) show stronger tendency to accumulate and cling to the riverbanks. As a result, the results in both conditions with vegetation and without vegetation in Figure 6 show more differences than Figure 5. Therefore, it can be concluded that factors that govern the movement of the floating objects are the size and the shape of the objects and vegetation. Therefore, from the result in Figure 5 and Figure 6 conclusions can be made that existence of vegetation seems to alter the flow of the river. Floating bodies with larger size have more influence from the river flow compared with small particles. [17]'s work, result shows different behaviour of flow between vegetation and non-vegetation channel. As a summary, introduction of vegetation will reduce cross-sectional velocity in magnitude while evolution pattern changes in crosssectional velocity with absence of vegetation. Presence of vegetation tends to stabilize position of the core of highest velocity so that it is maintained at a distance away from the outer bank around the bend [15]. Other researchers also came to a conclusion that inside the channel bend with high curvature downstream velocity take low values near the outer bank [18]. [19] also suggested that presence of vegetation would cause decrease in flow velocity thus improving the bank stability [19].

Several problem and limitations are yet to be discovered. Firstly, several researcher point out that although substantial research been carried out to discoverer the interaction between flow and vegetation, but not much systematic effort has been made to analyse flow-vegetation interaction in curved flows [15], therefore the reference regarding to this issue will be limited. Second, although detail information of variables change and flow characteristic can be represented in LES simulation method, but there is still limited. spacing between horizontal grid and vertical grid for computation are $25 \mathrm{~m}$ horizontal and $20 \mathrm{~cm}$ in vertical. More computational power is needed to run the simulation in a more 
detail way. Third, topography of the riverbed will be changed over time due to the transportation of the sediment; therefore, it will be a challenge to simulate the real time situation of the bathometry in the river. Finally, the way to model the resistance force created by the vegetation is a important during the simulation process.

\section{Conclusion}

The computation of solid bodies floating on the free surface such as the tree debris, has been extended to include the effects of the riparian vegetation. The whole method is based on the three dimensional LES with dynamically moving free surface. Tracking of the floating objects in real meandering river in Malaysia has been done using this method. While the effects of the vegetation appears significant, the locations of the vegetation are in the low speed and shallow areas. The present results indicate that the effects on the small objects are small but the large objects with long bar-like bodies are influenced by the local retardation due to the vegetation. The simulation of the large discharge still need to be done to anticipate the situation that will occur in a large flood occurring once in tens of years.

The present work was conducted with a partial support of Universiti Tunku Abdul Rahman Research Fund UTARRF 2016.

\section{References}

1. A. Hribernik, M. Fike and T.M. Hribernik, Journal of Trends in the Development of Machinery and Associated Technology, 17(1) 161-164 (2013)

2. F. Boogaard, Z. Vojinovic, Y.-C. Chen, J. Kluck and T.-P. Lin, Proc. International Symposium on Civil and Environmental Engineering, paper ICSEWR-039 (2016)

3. A. Nakayama, S. Chai, H. Goh, J. JSCE Ser B1 (Hydraulic Engineering), 74(4) I_761I_756 (2018)

4. A. Tominaga, M. Nagao and I. Nezu, Proc. 2nd International Symposium on Environmental Hydraulics, 329-334, (1998)

5. K. Blanckaert and W.H. Graf, J.Hydr. Eng. 130(3) 186-198 (2004)

6. C.-L. Jang and Y. Shimizu, J. Hydr. Res., 45(6) 763-772 (2007)

7. A. Nakayama and N. Hisasue, J. Hydr Res. 48(4) 415-427 (2010)

8. A. Nakayama and S. Yokojima, Proc. of Hydraulic Engineering, 46 373-378 (2002)

9. T.S. Lund,T.S., X. Wu, K.D. Squires, J. Comp. Phys., 140, 233-258 (1998)

10. R. Wilson and R. Shaw, J. Applic. Met., 116(11) 1197- 1205 (1977)

11. H.M. Nepf, J. Hydr Res. 50(3) 262-278 (2012)

12. D.R. Dilen, D.R., J. Forensic Sci. 29(4) 1027-37 (1984)

13. I. Kimura, Proc. 4th International Symposium on Shallow Flows, Eindhoven, Netherland (2017)

14. T. Kang, I. Kimura and Y. Shimizu, J. JSCE Ser B1 (Hydraulic Engineering) 74(4) I_757-I_762(2018)

15. D. Termini, J. Hydr. Res. 55(3) 423-429 (2017)

16. H.Schlichting, Boundary-layer theory, Springer (2017)

17. D.Termini, M. Piraino, ESPL. 36(2) 244-256 (2011)

18. S. D. Thorne, D.J. Furbish, Geomorphology 12 241-257 (1995) 
19. F. Lopez, M.Garcia, Water Resour. Res. 34 2341-2352 (1998) 